\title{
REDUCTION OF DIFFERENTIABLE EQUATIONS WITH IMPULSE EFFECT
}

\author{
LELDE SERMONE 1 \\ Latvian Academy of Sciences and University of Latvia \\ Institute of Mathematics \\ Turgeneva ieta 19, Riga, LV-1524, Latvia \\ e-mail: lab0098@lvrulv11.lanet.lv
}

(Received January, 1995; Revised December, 1995)

We consider a problem of a partial linearization of noninvertible differential equations with impulse effect and establish sufficient conditions for the dynamical equivalence.

Key words: Dynamical Equivalence, Noninvertible Impulse System.

AMS subject classifications: $34 \mathrm{~A} 37,34 \mathrm{C} 20$.

\section{Introduction}

The theory of differential equations with impulse effect has been developing rapidly in the recent years. These equations are convenient for description of evolutionary processes suddenly changing their state at certain moments. For the general theory of impulse systems, the reader is referred to the monographs by V. Lakshmikantham, D.D. Bainov and P.S. Simeonov [7], D.D. Bainov and P.S. Simeonov [2, 3, 5], D.D. Bainov and V. Covachev [4] and A.M. Samoilenko and N.A. Perestjuk [10]. The classification problems of impulse systems was first considered in [9] and [11]. This article is concerned with a specific aspect of classification, that reduces a noninvertible impulse system to a simpler one. Sufficient conditions for the dynamical equivalence of noninvertible differential systems are established. There are extensive works on classification for ordinary differential equations and maps $[1,6,8]$.

\section{The Statement of the Problem}

Let $\mathbf{X}$ and $\mathbf{Y}$ be Banach spaces. The norms in these spaces are denoted by $|\cdot|$. Consider the following system of differential equations with impulse effect at fixed moments:

\footnotetext{
${ }^{1}$ This work has been performed with the financial support of Latvian Council of Science under Grant 93.809.
} 


$$
\left\{\begin{array}{c}
d x / d t=A(t) x+f(t, x, y) \\
d y / d t=B(t) y+g(t, x, y) \\
\left.\Delta x\right|_{t=\tau_{i}}=C_{i} x\left(\tau_{i}-0\right)+I_{i}\left(x\left(\tau_{i}-0\right), y\left(\tau_{i}-\right)\right) \\
\left.\Delta y\right|_{t=\tau_{i}}=D_{i} y\left(\tau_{i}-0\right)+K_{i}\left(x\left(\tau_{i}-0\right), y\left(\tau_{i}-0\right)\right)
\end{array}\right.
$$

where:

(i) the maps $A: \mathbf{R} \rightarrow \operatorname{Hom}(\mathbf{X}), B: \mathbf{R} \rightarrow \operatorname{Hom}(\mathbf{Y})$ are locally integrable in Bochner's sense, where $\operatorname{Hom}(\mathbf{X})$ is the set of all linear bounded maps from $\mathbf{X}$ to $\mathbf{X}$;

(ii) the maps $f: \mathbf{R} \times \mathbf{X} \times \mathbf{Y} \rightarrow \mathbf{X}, g: \mathbf{R} \times \mathbf{X} \times \mathbf{Y} \rightarrow \mathbf{Y}$ are locally integrable in Bochner's sense with respect to $t$ for fixed $x$ and $y$ and they satisfy the Lipschitz conditions with small $\epsilon$ uniformly with respect to $t$ also:

$$
\begin{aligned}
& \left|f(t, x, y)-f\left(t, x^{\prime}, y^{\prime}\right)\right| \leq \epsilon\left(\left|x-x^{\prime}\right|+\left|y-y^{\prime}\right|\right) \\
& \left|g(t, x, y)-g\left(t, x^{\prime}, y^{\prime}\right)\right| \leq \epsilon\left(\left|x-x^{\prime}\right|+\left|y-y^{\prime}\right|\right)
\end{aligned}
$$

and, in addition

$$
\sup _{t, x, y}|f(t, x, y)|<+\infty
$$

(iii) $C_{i} \in \operatorname{Hom}(\mathbf{X}), D_{i} \in \operatorname{Hom}(\mathbf{Y})$ for $i \in \mathbf{N}$ and the maps $I_{i}: \mathbf{X} \times \mathbf{Y} \rightarrow \mathbf{X}, K_{i}: \mathbf{X} \times \mathbf{Y} \rightarrow \mathbf{Y}$ satisfy the Lipschitz conditions with small $\epsilon$ :

$$
\begin{gathered}
\left|I_{i}(x, y)-I_{i}\left(x^{\prime}, y^{\prime}\right)\right| \leq \epsilon\left(\left|x-x^{\prime}\right|+\left|y-y^{\prime}\right|\right) \\
\left|K_{i}(x, y)-K_{i}\left(x^{\prime}, y^{\prime}\right)\right| \leq \epsilon\left(\left|x-x^{\prime}\right|+\left|y-y^{\prime}\right|\right)
\end{gathered}
$$

and, in addition

$$
\sup _{i, x, y}\left|I_{i}(x, y)\right|<+\infty
$$

(iv) the moments $\tau_{i}$ of impulse effect form a strictly increasing sequence and

$$
\lim _{n \rightarrow \infty} \tau_{n}=+\infty
$$

Let $\Phi\left(\cdot, t_{0}, x_{0}, y_{0}\right):\left[t_{0},+\infty\right) \rightarrow \mathbf{X} \times \mathbf{Y}$ be a solution of the system (1), where $\Phi\left(t_{0}+0, t_{0}, x_{0}, y_{0}\right)=\left(x_{0}, y_{0}\right)$ and $\Phi\left(t, t_{0}, x_{0}, y_{0}\right)=\left(x\left(t, t_{0}, x_{0}, y_{0}\right), y\left(t, t_{0}, x_{0}, y_{0}\right)\right)$. At the break points, the values are taken at $\tau_{i}-0$, unless it is specified otherwise. Let $U(t, \tau)$ and $V(t, \tau)$ be the Cauchy evolution maps of the corresponding linear systems with impulse effect:

$$
\begin{gathered}
\left\{\begin{array}{c}
d U / d t=A(t) U, \\
\left.\Delta U\right|_{t=\tau_{i}}=C_{i} U\left(\tau_{i}-0\right),
\end{array}\right. \\
\left\{\begin{array}{c}
d V / d t=B(t) V, \\
\left.\Delta V\right|_{t=\tau_{i}}=D_{i} V\left(\tau_{i}-0\right) .
\end{array}\right.
\end{gathered}
$$

In addition, we assume that they satisfy the following inequalities: 


$$
\begin{gathered}
L=\sup _{t}\left(\int_{t}^{+\infty}|U(t, \tau)||V(\tau, t)| d \tau+\sum_{t \leq \tau_{i}}\left|U\left(t, \tau_{i}\right)\right|\left|V\left(\tau_{i}-0, t\right)\right|\right)<\infty, \\
M=\sup _{t}\left(\int_{t}^{+\infty}|U(t, \tau)| d \tau+\sum_{t \leq \tau_{i}}\left|U\left(t, \tau_{i}\right)\right|\right)<\infty .
\end{gathered}
$$

Let us consider (1) and the system

$$
\left\{\begin{array}{c}
d x / d t=A(t) x, \\
d y / d t=B(t) y+g(t, x+v(t, x, y), y), \\
\left.\Delta x\right|_{t=\tau_{i}}=C_{i} x\left(\tau_{i}-0\right), \\
\left.\Delta y\right|_{t=\tau_{i}}=D_{i} y\left(\tau_{i}-0\right)+K_{i}\left(x\left(\tau_{i}-0\right)\right. \\
\left.+v\left(\tau_{i}-0, x\left(\tau_{i}-0\right), y\left(\tau_{i}-0\right)\right), y\left(\tau_{i}-0\right)\right) .
\end{array}\right.
$$

Definition: Two systems of differential equations with impulse effect (1) and (4) are dynamically equivalent if there exists a map $H: \mathbf{R} \times \mathbf{X} \times \mathbf{Y} \rightarrow \mathbf{X} \times \mathbf{Y}$ with the following properties:

(i) $\quad H(t, \cdot, \cdot): \mathbf{X} \times \mathbf{Y} \rightarrow \mathbf{X} \times \mathbf{Y}$ is homeomorphism;

(ii) $H\left(t, \Phi\left(t, t_{0}, x_{0}, y_{0}\right)\right)=\Psi\left(t, t_{0}, H\left(t_{0}, x_{0}, y_{0}\right)\right)$, if $t \geq t_{0}$, where $\Psi:\left[t_{0},+\infty\right) \rightarrow \mathbf{X} \times \mathbf{Y}$, $\Psi\left(t, t_{0}, x_{0}, y_{0}\right)=\left(x_{0}\left(t, t_{0}, x_{0}, y_{0}\right), y_{0}\left(t, t_{0}, x_{0}, y_{0}\right)\right)$ is solution of the system (4);

(iii) if the system (1) is autonomous and without impulses, then $H$ does not depend on $t$.

\section{The Main Theorem}

Theorem 1: Let hypothesis (i)-(iv) be satisfied, and suppose the inequalities $4 L \epsilon \leq 1$ and $2 M \epsilon<1+\sqrt{1-4 L \epsilon}$ are satisfied, where the constants $L$ and $M$ are specified by formulas (2) and (3).

Then (1) and (4) are dynamically equivalent.

Proof: Step 1: Let us consider the Banach space $\mathbf{B}_{1}$ of the bounded maps that are continuous for $(t, x, y) \in\left(\tau_{i}, \tau_{i+1}\right] \times \mathbf{X} \times \mathbf{Y}$ and have first kind breaks for $t=\tau_{i}$ :

$$
\mathbf{B}_{1}=\left\{v \mid v: \mathbf{R} \times \mathbf{X} \times \mathbf{Y} \rightarrow \mathbf{X} \text { and } \sup _{t, x, y}|v(t, x, y)|<\infty\right\}
$$

with the norm $\|v\|=\sup _{t, x, y}|v(t, x, y)|$.

Let us define the set:

$$
\mathbf{G}_{1}(p)=\left\{v \in \mathbf{B}_{1}|| v(t, x, z)-v\left(t, x, z^{\prime}\right)|\leq p| z-z^{\prime} \mid\right\} .
$$

$\mathbf{G}_{1}(p)$ is a closed subset of the Banach space $\mathbf{B}_{1}$. In $\mathbf{G}_{1}(p)$ we consider the functional equation:

$$
\begin{aligned}
& v_{1}\left(t_{0}, x_{0}, z\right)=-\int_{t_{0}}^{+\infty} U\left(t_{0}, \tau\right) f\left(\tau, x_{0}(\tau)+v_{1}\left(\tau, x_{0}(\tau), z(\tau)\right), z(\tau)\right) d \tau \\
& -\sum_{t_{0} \leq \tau_{1}} U\left(t_{0}, \tau_{i}\right) I_{i}\left(x_{0}\left(\tau_{i}-0\right)+v_{1}\left(\tau_{i}-0, x_{0}\left(\tau_{i}-0\right), z\left(\tau_{i}-0\right)\right), z\left(\tau_{i}-0\right)\right)
\end{aligned}
$$


and

$$
\begin{gathered}
z(t)=V\left(t, t_{0}\right) z+\int_{t_{0}}^{t} V(t, \tau) g\left(\tau, x_{0}(\tau)+v_{1}\left(\tau, x_{0}(\tau), z(\tau)\right), z(\tau)\right) d \tau \\
+\sum_{t_{0} \leq t} V\left(t, \tau_{i}\right) K_{i}\left(x_{0}\left(\tau_{i}-0\right)+v_{1}\left(\tau_{i}-0, x_{0}\left(\tau_{i}-0\right), z\left(\tau_{i}-0\right)\right), z\left(\tau_{i}-0\right)\right)
\end{gathered}
$$

where $x_{0}(\tau)=U\left(\tau, t_{0}\right) x_{0}$.

To solve the functional equation (6) we introduce the operator $\mathbf{E}$ from $\mathbf{G}_{1}(p)$ to $\mathbf{B}_{1}$ by the formula:

$$
\begin{aligned}
& \mathbf{E} v_{1}\left(t_{0}, x_{0}, z\right)=-\int_{t_{0}}^{+\infty} U\left(t_{0}, \tau\right) f\left(\tau, x_{0}(\tau)+v_{1}\left(\tau, x_{0}(\tau), z(\tau)\right), z(\tau)\right) d \tau \\
& -\sum_{t_{0} \leq \tau_{1}} U\left(t_{0}, \tau_{i}\right) I_{i}\left(x_{0}\left(\tau_{i}-0\right)+v_{1}\left(\tau_{i}-0, x_{0}\left(\tau_{i}-0\right), z\left(\tau_{i}-0\right)\right), z\left(\tau_{i}-0\right)\right)
\end{aligned}
$$

and

$$
\begin{gathered}
z(t)=V\left(t, t_{0}\right) z+\int_{t_{0}}^{t} V(t, \tau) g\left(\tau, x_{0}(\tau)+v_{1}\left(\tau, x_{0}(\tau), z(\tau)\right), z(\tau)\right) d \tau \\
+\sum_{t_{0} \leq t} V\left(t, \tau_{i}\right) K_{i}\left(x_{0}\left(\tau_{i}-0\right)+v_{1}\left(\tau_{i}-0, x_{0}\left(\tau_{i}-0\right), z\left(\tau_{i}-0\right)\right), z\left(\tau_{i}-0\right)\right) .
\end{gathered}
$$

Next, we determine the difference $\left\|\mathbf{E} v_{1}\left(t_{0}, x_{0}, z\right)-\mathbf{E} v_{1}^{\prime}\left(t_{0}, x_{0}, z^{\prime}\right)\right\|$, taking into account the properties of $f, I_{i}, v_{1}$. We obtain that:

$$
\begin{gathered}
\left\|\mathbf{E} v_{1}\left(t_{0}, x_{0}, z\right)-\mathbf{E} v_{1}^{\prime}\left(t_{0}, x_{0}, z^{\prime}\right)\right\| \leq \epsilon(p+1)\left(\int_{t_{0}}^{+\infty}\left|U\left(t_{0}, \tau\right)\right|\left|z(\tau)-z^{\prime}(\tau)\right| d \tau\right. \\
\left.\quad+\sum_{t_{0} \leq \tau_{i}}\left|U\left(t_{0}, \tau_{i}\right)\right|\left|z\left(\tau_{i}-0\right)-z^{\prime}\left(\tau_{i}-0\right)\right|\right)+\epsilon M\left\|v_{1}-v_{1}^{\prime}\right\| .
\end{gathered}
$$

On the other hand, we estimate the difference $\left|z(t)-z^{\prime}(t)\right|$, taking into consideration the properties of $g$ and $K_{i}$ :

$$
\begin{aligned}
\left|z(t)-z^{\prime}(t)\right| \leq\left|V\left(t, t_{0}\right)\right|\left|z-z^{\prime}\right|+\epsilon(p+1)\left(\int_{t_{0}}^{t}|V(t, \tau)|\left|z(\tau)-z^{\prime}(\tau)\right| d \tau\right. \\
\left.\quad+\sum_{t_{0} \leq \tau_{i}}\left|V\left(t, \tau_{i}\right)\right|\left|z\left(\tau_{i}-0\right)-z^{\prime}\left(\tau_{i}-0\right)\right|\right) \\
+\epsilon\left\|v_{1}-v_{1}^{\prime}\right\|\left(\int_{t_{0}}^{t}|V(t, \tau)| d \tau+\sum_{t_{0} \leq t}\left|V\left(t, \tau_{i}\right)\right|\right) .
\end{aligned}
$$

Multiplying the difference $\left|z(t)-z^{\prime}(t)\right|$ by $\left|U\left(t_{0}, t\right)\right|$ and integrating from $t_{0}$ to $+\infty$, we obtain:

$$
\int_{t_{0}}^{+\infty}\left|U\left(t_{0}, t\right)\right|\left|z(t)-z^{\prime}(t)\right| d t \leq\left|z-z^{\prime}\right| \int_{t_{0}}^{+\infty}\left|U\left(t_{0}, t\right)\right|\left|V\left(t, t_{0}\right)\right| d t
$$




$$
\begin{aligned}
& +\sup _{\tau} \int_{t_{0}}^{+\infty}|U(\tau, t)||V(t, \tau)| d t\left(\epsilon ( p + 1 ) \left(\int_{t_{0}}^{+\infty}\left|U\left(t_{0}, \tau\right)\right|\left|z(\tau)-z^{\prime}(\tau)\right| d \tau\right.\right. \\
& \left.\quad+\sum_{t_{0} \leq \tau_{i}}\left|U\left(t_{0}, \tau_{i}\right)\right|\left|z\left(\tau_{i}-0\right)-z^{\prime}\left(\tau_{i}-0\right)\right|\right) \\
& \left.+\epsilon\left\|v_{1}-v_{1}^{\prime}\right\|\left(\int_{t_{0}}^{+\infty}\left|U\left(t_{0}, \tau\right)\right| d \tau+\sum_{t_{0} \leq \tau_{i}}\left|U\left(t_{0}, \tau_{i}\right)\right|\right)\right)
\end{aligned}
$$

Multiplying the same difference by $\left|U\left(t_{0}, \tau_{i}\right)\right|$ and summing for all $i$ with respect to $t_{0} \leq \tau_{i}$, we get:

$$
\begin{gathered}
\sum_{t_{0} \leq \tau_{i}}\left|U\left(t_{0}, \tau_{i}\right)\right|\left|z\left(\tau_{i}-0\right)-z^{\prime}\left(\tau_{i}-0\right)\right| \\
\leq\left|z-z^{\prime}\right| \sum_{t_{0} \leq \tau_{i}}\left|U\left(t_{0}, \tau_{i}\right)\right|\left|V\left(\tau_{i}-0, t_{0}\right)\right| \\
+\sup _{\tau_{t_{0}} \leq \tau_{i}}\left|U\left(\tau, \tau_{i}\right)\right|\left|V\left(\tau_{i}-0, \tau\right)\right|\left(\epsilon ( p + 1 ) \left(\int_{t_{0}}^{+\infty}\left|U\left(t_{0}, \tau\right)\right|\left|z(\tau)-z^{\prime}(\tau)\right| d \tau\right.\right. \\
\left.+\sum_{t_{0} \leq \tau_{i}}\left|U\left(t_{0}, \tau_{i}\right)\right|\left|z\left(\tau_{i}-0\right)-z^{\prime}\left(\tau_{i}-0\right)\right|\right) \\
+\epsilon\left\|v_{1}-v_{1}^{\prime}\right\|\left(\int_{t_{0}}^{+\infty}\left|U\left(t_{0}, \tau\right)\right| d \tau+\sum_{t_{0} \leq \tau_{i}}\left|U\left(t_{0}, \tau_{i}\right)\right|\right)
\end{gathered}
$$

Summing up (8) and (9) we get:

$$
\begin{aligned}
& \int_{t_{0}}^{+\infty}\left|U\left(t_{0}, \tau\right)\right|\left|z(t)-z^{\prime}(t)\right| d \tau+\sum_{t_{0} \leq \tau_{i}}\left|U\left(t_{0}, \tau_{i}\right)\right|\left|z\left(\tau_{i}-0\right)-z^{\prime}\left(\tau_{i}-0\right)\right| \\
& \quad \leq L\left(\left|z-z^{\prime}\right|+\epsilon(p+1)\left(\int_{t_{0}}^{+\infty}\left|U\left(t_{0}, \tau\right)\right|\left|z(\tau)-z^{\prime}(\tau)\right| d \tau\right.\right. \\
& \left.\left.\quad+\sum_{t_{0} \leq \tau_{i}}\left|U\left(t_{0}, \tau_{i}\right)\right|\left|z\left(\tau_{i}-0\right)-z^{\prime}\left(\tau_{i}-0\right)\right|\right)+\epsilon M\left\|v_{1}-v_{1}^{\prime}\right\|\right) .
\end{aligned}
$$

Let us designate

$$
\int_{t_{0}}^{+\infty}\left|U\left(t_{0}, \tau\right)\right|\left|z(\tau)-z^{\prime}(\tau)\right| d t+\sum_{t_{0} \leq \tau_{i}}\left|U\left(t_{0}, \tau_{i}\right)\right|\left|z\left(\tau_{i}-0\right)-z^{\prime}\left(\tau_{i}-0\right)\right|=Q .
$$


We suppose that

Inequality (10) means:

$$
\epsilon(p+1) L<1
$$

$$
Q \leq L\left(\left|z-z^{\prime}\right|+\epsilon(p+1) Q+\epsilon M\left\|v_{1}-v_{1}^{\prime}\right\|\right) .
$$

Thus we have shown that

$$
Q \leq \frac{L\left(\left|z-z^{\prime}\right|+\epsilon M\left\|v_{1}-v_{1}^{\prime}\right\|\right)}{1-\epsilon L(p+1)} .
$$

Taking into consideration inequalities (7) and (10), we get

$$
\begin{gathered}
\left\|\mathbf{E} v_{1}\left(t_{0}, x_{0}, z\right)-\mathbf{E} v_{1}^{\prime}\left(t_{0}, x_{0}, z^{\prime}\right)\right\| \\
\leq \epsilon(p+1) \frac{L\left(\left|z-z^{\prime}\right|+\epsilon M\left\|v_{1}-v_{1}^{\prime}\right\|\right)}{1-\epsilon L(p+1)}+\epsilon M\left\|v_{1}-v_{1}^{\prime}\right\| .
\end{gathered}
$$

If $4 L \epsilon \leq 1$, then there is $p>0$ satisfying

$$
\frac{\epsilon L(p+1)}{1-\epsilon L(p+1)} \leq p \text {. }
$$

We choose

$$
p=\frac{1-2 \epsilon L-\sqrt{1-4 \epsilon L}}{2 \epsilon L}
$$

Then,

$$
p+1=\frac{1-\sqrt{1-4 \epsilon L}}{2 \epsilon L}
$$

and $2 \epsilon L(p+1) \leq 1$. We insert this $p$ into (11) and obtain

$$
\left\|\mathbf{E} v_{1}\left(t_{0}, x_{0}, z\right)-\mathbf{E} v_{1}^{\prime}\left(t_{0}, x_{0}, z^{\prime}\right)\right\| \leq p\left|z-z^{\prime}\right|+\epsilon M(p+1)\left\|v_{1}-v_{1}^{\prime}\right\| .
$$

If $2 \epsilon M<1+\sqrt{1-4 \epsilon L}$, then $\epsilon M(p+1)<1$. We conclude that $\mathbf{E}$ is a contraction. It shows that there is only one solution in $\mathbf{G}_{1}(p)$, satisfying the functional equation (6).

Next, we construct the map:

$$
H_{1}\left(t_{0}, x_{0}, z\right)=\left(h_{1}\left(t_{0}, x_{0}, z\right), z\right)=\left(x_{0}+v_{1}\left(t_{0}, x_{0}, z\right), z\right) .
$$

It can easily checked that:

$$
H_{1}\left(t, \Psi\left(t, t_{0}, x_{0}, y_{0}\right)\right)=\Phi\left(t, t_{0}, H_{1}\left(t_{0}, x_{0}, y_{0}\right)\right)
$$

$\underline{\text { Step 2: }}$ In the same space $\mathrm{G}_{1}(p)$, we define the map by the following formula:

$$
\begin{gathered}
v_{2}\left(t_{0}, x_{0}, y_{0}\right)=\int_{t_{0}}^{+\infty} U\left(t_{0}, \tau\right) f\left(\tau, \Phi\left(\tau, t_{0}, x_{0}, y_{0}\right)\right) d \tau \\
\quad+\sum_{t_{0} \leq \tau_{i}} U\left(t_{0}, \tau_{i}\right) I_{i}\left(\Phi\left(\tau_{i}-0, t_{0}, x_{0}, y_{0}\right)\right)
\end{gathered}
$$


Next, we compute that

$$
\begin{gathered}
v_{2}\left(t, \Phi\left(t, t_{0}, x_{0}, y_{0}\right)\right)=\int_{t}^{+\infty} U(t, \tau) f\left(\tau, \Phi\left(\tau, t_{0}, x_{0}, y_{0}\right)\right) d \tau \\
\quad+\sum_{t \leq \tau_{i}} U\left(t, \tau_{i}\right) I_{i}\left(\Phi\left(\tau_{i}-0, t_{0}, x_{0}, y_{0}\right)\right) .
\end{gathered}
$$

We construct the map:

$$
H_{2}\left(t_{0}, x_{0}, y_{0}\right)=\left(h_{2}\left(t_{0}, x_{0}, y_{0}\right), y_{0}\right)=\left(x_{0}+v_{2}\left(t_{0}, x_{0}, y_{0}\right), y_{0}\right) .
$$

Applying (12), we conclude that

$$
h_{2}\left(t, \Phi\left(t, t_{0}, x_{0}, y_{0}\right)\right)=U\left(t, t_{0}\right) h_{2}\left(t_{0}, x_{0}, y_{0}\right)
$$

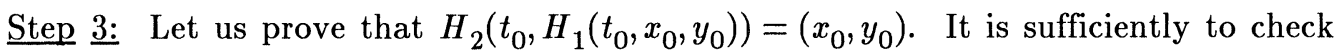
that

$$
\begin{gathered}
h_{2}\left(t_{0}, H_{1}\left(t_{0}, x_{0}, y_{0}\right)\right)=h_{1}\left(t_{0}, x_{0}, y_{0}\right)+v_{2}\left(t_{0}, H_{1}\left(t_{0}, x_{0}, y_{0}\right)\right) \\
=x_{0}+v_{1}\left(t_{0}, x_{0}, y_{0}\right)+v_{2}\left(t_{0}, H_{1}\left(t_{0}, x_{0}, y_{0}\right)\right) \\
=x_{0}-\int_{t_{0}}^{+\infty} U\left(t_{0}, \tau\right) f\left(\tau, \Phi\left(\tau, t_{0}, H_{1}\left(t_{0}, x_{0}, y_{0}\right)\right)\right) d \tau \\
\quad-\sum_{t_{0} \leq \tau_{i}} U\left(t_{0}, \tau_{i}\right) I_{i}\left(\Phi\left(\tau_{i}-0, t_{0}, H_{1}\left(t_{0}, x_{0}, y_{0}\right)\right)\right) \\
+\int_{t_{0}}^{+\infty} U\left(t_{0}, \tau\right) f\left(\tau, \Phi\left(\tau, t_{0}, H_{1}\left(t_{0}, x_{0}, y_{0}\right)\right)\right) d \tau \\
+\sum_{t_{0} \leq \tau_{i}} U\left(t_{0}, \tau_{i}\right) I_{i}\left(\Phi\left(\tau_{i}-0, t_{0}, H_{1}\left(t_{0}, x_{0}, y_{0}\right)\right)\right)=x_{0}
\end{gathered}
$$

Step 4: Now let us prove $H_{1}\left(t_{0}, H_{2}\left(t_{0}, x_{0}, y_{0}\right)\right)=\left(x_{0}, y_{0}\right)$. Let us consider the Banach space $\mathbf{B}_{2}$ of the bounded maps that are continuous for $(t, x, y, z) \in\left(\tau_{i}, \tau_{i+1}\right] \times \mathbf{X} \times$ $\mathbf{Y} \times \mathbf{Z}$ and have first kind breaks for $t=\tau_{i}$ :

$$
\mathbf{B}_{2}=\left\{v \mid v: \mathbf{R} \times \mathbf{X} \times \mathbf{Y} \times \mathbf{Z} \rightarrow \mathbf{X} \text { and } \sup _{t, x, y, z}|v(t, x, y, z)|<\infty\right\}
$$

with the norm $\|v\|=\sup _{t, x, y, z}|v(t, x, y, z)|$.

Let us introduce the set:

$$
\mathbf{G}_{2}(p)=\left\{v \in \mathbf{B}_{2}|| v\left(t_{0}, x, y, z\right)-v\left(t_{0}, x, y, z^{\prime}\right)|\leq p| z-z^{\prime} \mid\right\} .
$$

In the space $\mathbf{G}_{2}(p)$ we consider the functional equation:

$$
v_{3}\left(t_{0}, x_{0}, y_{0}, z\right)=\int_{t_{0}}^{+\infty} U\left(t_{0}, \tau\right)\left(f(\tau, \Phi(\tau))-f\left(\tau, x(\tau)+v_{3}(\tau, \Phi(\tau), z(\tau)), z(\tau)\right) d \tau\right.
$$




$$
\begin{gathered}
+\sum_{t_{0}<\tau_{i}} U\left(t_{0}, \tau_{i}\right)\left(I_{i}\left(\Phi\left(\tau_{i}-0\right)\right)\right. \\
\left.-I_{i}\left(x\left(\tau_{i}-0\right)+v_{3}\left(\tau_{i}-0, \Phi\left(\tau_{i}-0\right), z\left(\tau_{i}-0\right)\right), z\left(\tau_{i}-0\right)\right)\right)
\end{gathered}
$$

and

$$
\begin{gathered}
z(t)=V\left(t, t_{0}\right) z+\int_{t_{0}}^{t} V(t, \tau) g\left(\tau, x(\tau)+v_{3}(\tau, \Phi(\tau), z(\tau)), z(\tau)\right) d \tau \\
+\sum_{t_{0} \leq \tau_{i}} V\left(t, \tau_{i}\right) K_{i}\left(x\left(\tau_{i}-0\right)+v_{3}\left(\tau_{i}-0, \Phi\left(\tau_{i}-0\right), z\left(\tau_{i}-0\right)\right), z\left(\tau_{i}-0\right)\right) .
\end{gathered}
$$

We introduce the operator $\mathbf{E}$ :

$$
\begin{aligned}
\mathbf{E} v_{3}\left(t_{0}, x_{0}, y_{0}, z\right)= & \int_{t_{0}}^{+\infty} U\left(t_{0}, \tau\right)\left(f(\tau, \Phi(\tau))-f\left(\tau, x(\tau)+v_{3}(\tau, \Phi(\tau), z(\tau)), z(\tau)\right) d \tau\right. \\
& +\sum_{\tau_{0} \leq \tau_{i}} U\left(t_{0}, \tau_{i}\right)\left(I_{i}\left(\Phi\left(\tau_{i}-0\right)\right)-I_{i}\left(x\left(\tau_{i}-0\right)\right.\right. \\
& \left.\left.+v_{3}\left(\tau_{i}-0, \Phi\left(\tau_{i}-0\right), z\left(\tau_{i}-0\right)\right), z\left(\tau_{i}-0\right)\right)\right)
\end{aligned}
$$

In the same manner as we proceeded in the first step, we determine the difference $\left\|\mathbf{E} v_{3}-\mathbf{E} v_{3}^{\prime}\right\|$. We make the same decisions and finally obtain that $\mathbf{E}$ is a contraction in $\mathbf{G}_{2}(p)$. There is only one solution for the functional equation (14). Next, we construct the map:

$$
H_{3}\left(t_{0}, x_{0}, y_{0}, z\right)=\left(h_{3}\left(t_{0}, x_{0}, y_{0}, z\right), z\right)=\left(x_{0}+v_{3}\left(t_{0}, x_{0}, y_{0}, z\right), z\right) .
$$

We notice that the map

$$
\alpha\left(t_{0}, x_{0}, y_{0}, z\right)=v_{2}\left(t_{0}, x_{0}, y_{0}\right)+v_{1}\left(t_{0}, h_{2}\left(t_{0}, x_{0}, y_{0}\right), z\right)
$$

also satisfies the functional equation (14) and $\alpha \in \mathbf{G}_{2}(p)$, therefore

$$
v_{3}\left(t_{0}, x_{0}, y_{0}, z\right)=v_{2}\left(t_{0}, x_{0}, y_{0}\right)+v_{1}\left(t_{0}, h_{2}\left(t_{0}, x_{0}, y_{0}\right), z\right)
$$

Now we set equal the third and the fourth argument of $v_{3}$ and put them into the expression. We obtain $v_{3}\left(t_{0}, x_{0}, y_{0}, y_{0}\right)=0$. Therefore $H_{1}\left(t_{0}, H_{2}\left(t_{0}, x_{0}, y_{0}\right)\right)=$ $\left(x_{0}, y_{0}\right)$

$$
\begin{gathered}
h_{1}\left(t_{0}, H_{2}\left(t_{0}, x_{0}, y_{0}\right)\right)=h_{2}\left(t_{0}, x_{0}, y_{0}\right)+v_{1}\left(t_{0}, H_{2}\left(t_{0}, x_{0}, y_{0}\right)\right) \\
=x_{0}+v_{2}\left(t_{0}, x_{0}, y_{0}\right)+v_{1}\left(t_{0}, H_{2}\left(t_{0}, x_{0}, y_{0}\right)\right)=x_{0}
\end{gathered}
$$

We get that $H_{1}(t, \cdot, \cdot)$ is a homeomorphism establishing dynamical equivalence of systems (1) and (4). 


\section{Acknowledgement}

I would like to thank my advisor, Professor Dr. A. Reinfelds, for suggesting and discussing this problem.

\section{References}

[1] Aulbach, B. and Garay, B.M., Linearizing the expanding part of noninvertible mappings, Z. Angew. Math. Phys. 44:3 (1993), 469-494.

[2] Bainov, D.D. and Simeonov, P.S., Systems with Impulsive Effect: Stability, Theory and Applications, Ellis Horwood 1989.

[3] Bainov, D.D. and Simeonov, P.S., Theory of Impulsive Differential Equations: Periodic Solutions and Applications, Longman Science \& Tech., New York 1993.

[4] Bainov, D.D. and Simeonov, P.S., Impulsive Differential Equations with a Small Parameter, World Scientific Publishing, River Edge, NJ 1994.

[5] Bainov, D.D. and Simeonov, P.S., Theory of Impulsive Differential Equations: Asymptotic Properties of the Solutions and Applications, World Scientific Publishing Co., River Edge, NJ 1995.

[6] Kirchgraber, U. and Palmer, K.J., Geometry in the Neighborhood of Invariant Manifolds of Maps and Flows and Linearization, Pitman Res. Notes Math. Ser. 233, Longman Sci. \& Tech., New York 1990.

[7] Lakshmikantham, V., Bainov, D.D. and Simeonov, P.S., Theory of Impulsive Differential Equations, World Scientific Publishing Co., River Edge, NJ 1989.

[8] Reinfelds, A., A reduction theorem for extensions of dynamical systems, Latv. Math. Ezhegodnik 33 (1989), 67-75 (in Russian).

[9] Reinfelds, A. and Sermone, L., Equivalence of nonlinear differential equations with impulse effect in Banach space, Latv. Univ. Zin̄a tn. Raksti 577 (1992), 6873.

[10] Samoilenko, A.M. and Perestjuk, N.A., Differential Equations with Impulse Effect, Vishcha Shkola, Kiev, Russia 1987 (in Russian).

[11] Sermone, L., Equivalence of differential equations with impulse effect, Proc. Latvian Acad. Sci. Section B:2 (1994), 78-80. 


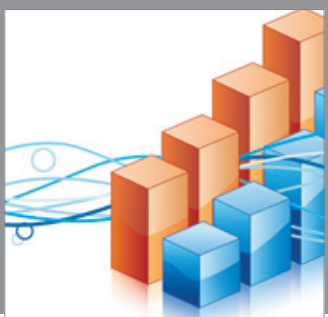

Advances in

Operations Research

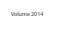

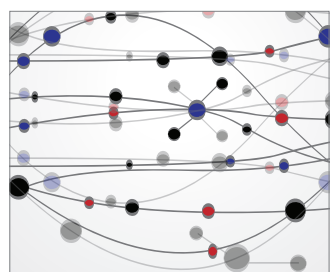

\section{The Scientific} World Journal
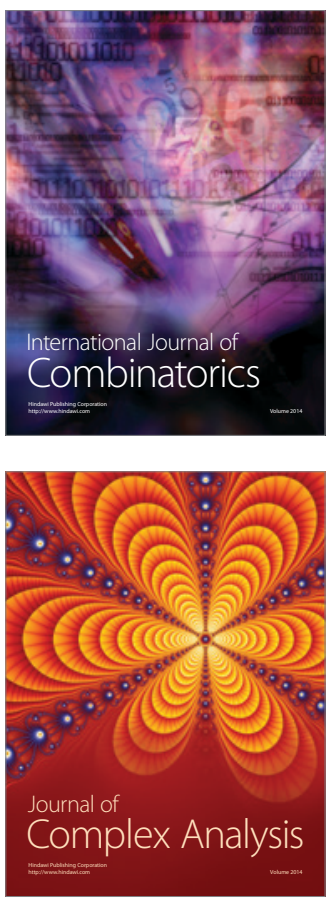

International Journal of

Mathematics and

Mathematical

Sciences
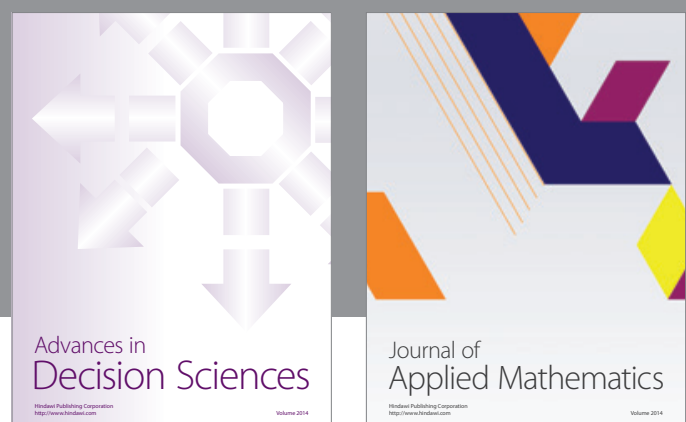

Journal of

Applied Mathematics
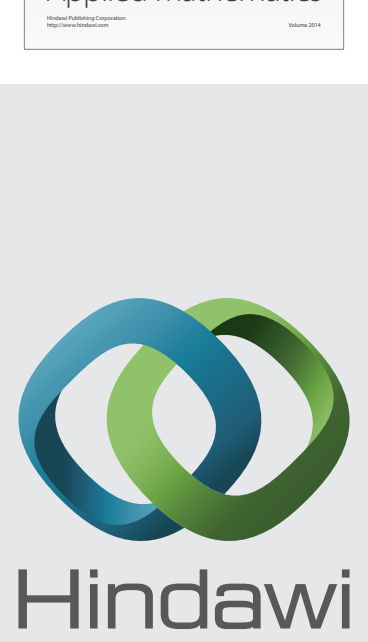

Submit your manuscripts at http://www.hindawi.com
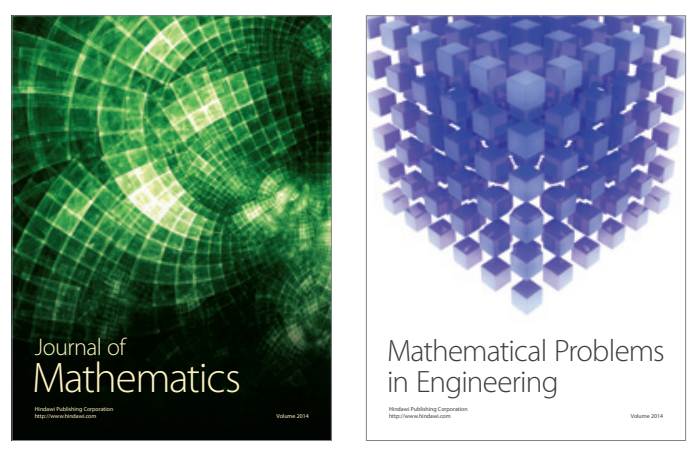

Mathematical Problems in Engineering
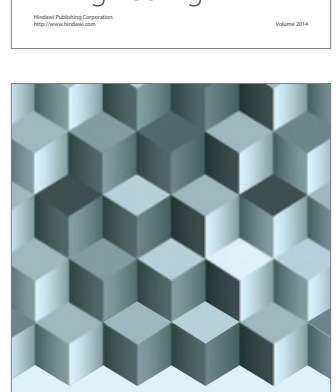

Journal of

Function Spaces
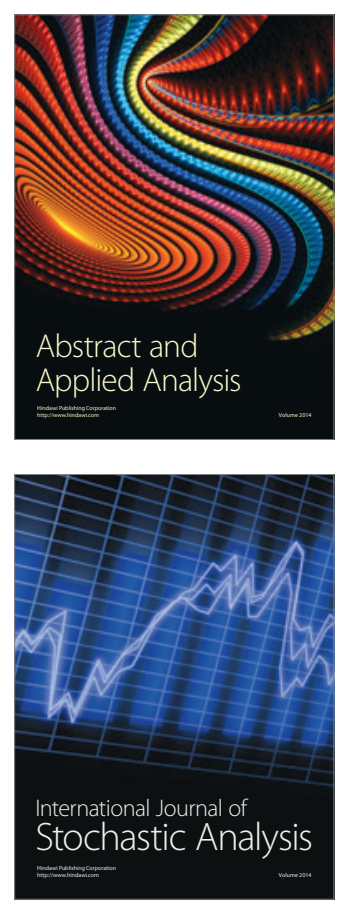

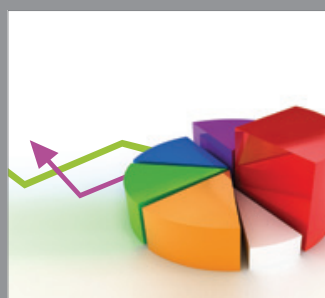

ournal of

Probability and Statistics

Promensencen
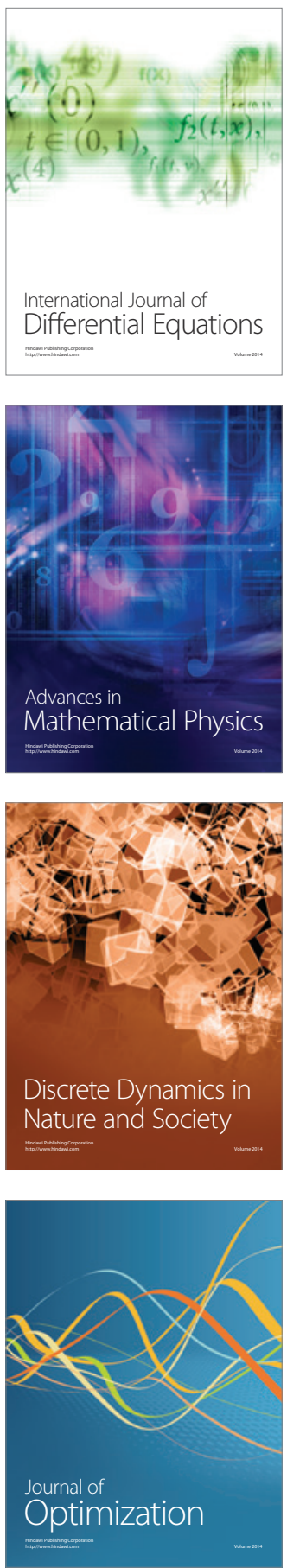